\title{
Social Media in Dermatology and an Overview of Popular Social Media Platforms
}

\author{
Mindy D. Szeto ${ }^{1} \cdot$ Andrina Mamo $^{1} \cdot$ Antara Afrin $^{2} \cdot$ Michelle Militello $^{3} \cdot$ Cara Barber $^{4}(\mathbb{0}$ \\ Accepted: 20 August 2021 / Published online: 19 October 2021 \\ ( $)$ The Author(s), under exclusive licence to Springer Science+Business Media, LLC, part of Springer Nature 2021
}

\begin{abstract}
Purpose of Review Social media provides an accessible and increasingly popular avenue for sharing healthcare information, networking, and outreach in dermatology. We provide an overview of prominent social media platforms, also known as applications or apps, as well as a discussion of their influence and implications for the field.

Recent Findings The various collaborative features of Facebook, Twitter, Instagram, TikTok, YouTube, Snapchat, and other emerging platforms have proven appealing to organizations and users seeking dermatology-related content and medical advice. However, the potential for propagation of inaccurate or even dangerous information is high.

Summary Despite the risks associated with social media usage, dermatology can benefit from opportunities to connect and engage with audiences through these platforms. Dermatologists should be encouraged to increase their presence on multiple social media apps to dispel and counteract misleading posts with evidence-based knowledge.
\end{abstract}

Keywords Social media applications $\cdot$ Facebook $\cdot$ Twitter $\cdot$ Instagram $\cdot$ TikTok $\cdot$ YouTube

\section{Introduction}

Social media has become an integral portion of society, with rising usage and prevalence since its creation and further expansion during the COVID-19 pandemic. The marked growth in social media was fueled by a large shift to all virtual platforms for human interactions. This is driven by increased public safety measures through widespread business and school closures, as well as lockdowns restricting public travel and large gatherings. These strict physical

This article is part of the Topical Collection on Dermatology and its Use in Social Media

Mindy D. Szeto and Andrina Mamoa are co-first authors.

Cara Barber

cara.barber@silverfallsderm.net

1 Department of Dermatology, University of Colorado Anschutz Medical Campus, Aurora, CO, USA

2 College of Human Medicine, Michigan State University, East Lansing, MI, USA

3 College of Osteopathic Medicine, Rocky Vista University, Parker, CO, USA

4 Department of Dermatology, Good Samaritan Regional Medical Center/Silver Falls Dermatology, Salem, OR, USA distancing measures caused people to be heavily reliant on virtual media, especially social media, for the latest pandemic news and to remain connected to each other [1]. Social media is broadly defined in this context as websites, or applications (apps), wherein the general public can create and distribute content, share photos and videos, and build social networks by interacting with other users in real time. In 2018, it was estimated that over 3 billion of the world's 7.7 billion population used at least one form of social media [2]. Additionally, the popularity of virtual communication through social media continues to grow dramatically [3].

Notably, social media has largely impacted medicine, with how healthcare providers, educators, patients, and peers interface, both in how medical knowledge is disseminated and care is delivered. Several popular apps have dominated social media in recent years, including Facebook, Twitter, Instagram, TikTok, YouTube, Snapchat, and others. Highly prolific and popular users with large social media followings known as "influencers" can often have an outsize impact on information transmitted through these platforms [4]. Especially in dermatology, providers have leveraged social media to directly connect with and educate the public, as well as promote their brand and practice. In fact, it has been suggested that dermatologists with a social media presence 
Table 1 This table is an abbreviated breakdown of the key social media platforms discussed, number of users, and most pertinent dermatology related content that can be found on each for quick reference

\begin{tabular}{|c|c|c|}
\hline $\begin{array}{l}\text { Social media } \\
\text { platform }\end{array}$ & Number of active users & Current dermatology-related uses on each application \\
\hline Facebook & 2.85 billion & $\begin{array}{l}\text {-Disease-specific support pages } \\
\text {-High impact journals sharing content } \\
\text {-Dermatology residency information }\end{array}$ \\
\hline Instagram & 1.074 billion & $\begin{array}{l}\text {-Disease-specific hashtags and discussion } \\
\text {-Photo/videos of dermatologic information and procedures } \\
\text {-High impact journals sharing content } \\
\text {-Product promotion } \\
\text {-Dermatology residency information }\end{array}$ \\
\hline Twitter & 199 million & $\begin{array}{l}\text {-Disease-specific support pages } \\
\text {-High impact journals sharing content } \\
\text {-Discussion of dermatology topics }\end{array}$ \\
\hline TikTok & 1 billion & $\begin{array}{l}\text {-Product promotion } \\
\text {-Photo/videos of dermatologic information and procedures }\end{array}$ \\
\hline YouTube & 2.1 billion & $\begin{array}{l}\text {-Product promotion } \\
\text {-Photo/videos of dermatologic information and procedures }\end{array}$ \\
\hline Snapchat & 280 million & -Photo/videos of dermatologic information and procedures \\
\hline Reddit & 430 million & -Disease-specific support pages \\
\hline
\end{tabular}

receive higher patient ratings [5]. In this narrative review, we provide an overview of each app, detailing unique features, popularity, influence, and current implementation in dermatology, also seen in Table 1 [6, 7, 19, 22, 29, 30, 43, 52, 55]. We hope to offer insight into both the benefits and potential pitfalls of social media usage as a guide for dermatology providers and patients alike.

\section{Facebook and Dermatology}

Created by Mark Zuckerberg in 2004, Facebook was the first social media platform ever to surpass 1 billion registered accounts. It currently has 2.85 billion monthly active users and 1.88 billion users that visit the social networking site daily [6]. Facebook has several functions available to its users, who can register to create their own profile, upload posts, pictures, videos, and send messages. With a customizable news feed, users can see posts from their friends and comment or react, such as "like" or "love." Facebook Groups centered around a shared interest can also be created by individual users, where group members have a space to post relevant content, such as events and media, and comment on or promote information. While some groups are open to the public to join at any time, some may be "closed," prompting users to request an invitation from group administrators. Facebook users can also engage with pages focused on their interests, learn of events happening around the world, or post ads and sell items to other users via Facebook Marketplace.

The dermatology field is expanding its social media presence, and Facebook is no exception. Influential academic journals, such as the Journal of American Academy of Dermatology (JAAD), have a high engagement rate on Facebook, owing to the large amount of likes and comments from users interested in newly published research [7]. Organizations such as the American Academy of Dermatology (AAD) have popular pages on Facebook to disseminate information, and as of July 16, 2021, the AAD page had 180,281 followers and 168,266 likes on Facebook [8]. In contrast, dermatology residency programs historically do not seem to be as highly involved in social media presence on Facebook, with only $23 \%$ having Facebook pages pre-COVID-19. This may have been due to the excess number of qualified candidates applying to enter the dermatology field and the presumed lack of necessity for increased social media attention [9]. Interestingly, many residency programs have increased their presence during the recent pandemic through Twitter and Instagram. Now, 53\% (74/140) of dermatology residency programs have an Instagram account as of 2020, with an $80 \%$ increase seen in 2020 alone and only a $14 \%$ increase in Facebook pages (21/140) [10]. This may continue to change in the future, as social media can play an important role in helping potential candidates learn more about the program's culture, promoting opportunities, and community outreach.

While Facebook demonstrates prominence among colleagues in academic dermatology, it also impacts how patients and the public perceive dermatology. There is evidence that Facebook is utilized more among patients in comparison with other social media platforms; in one study examining the effects of social media on melanoma screening clinic attendance, over $60 \%$ of the patient cohort reported using Facebook, as compared to only $28 \%$ and $12 \%$ reporting Instagram and Twitter use, respectively [11]. A 
possible explanation for this is due to the patient's ability to learn about different dermatologic diseases and find support systems through Facebook Groups. These disease-focused Facebook Groups are generally linked to keywords or diagnoses shared by patients with the same disease and are typically run by non-medical professionals. Facebook can be a supportive community for those with skin conditions, akin to traditional in-person support groups. Shared personal experiences help by providing psychosocial support, as well as posts offering medical advice, even though a majority of this advice may be unsubstantiated by evidence-based medicine [12]. Currently, the largest online support group for patients with hidradenitis suppurativa (HS) is on Facebook, and these spaces have allowed patients to assist each other with emotional challenges and other issues. In an analysis of the content of the largest group, the majority of HS group posts focused on social support (20\%) and requesting information (i.e., lifestyle issues or specific symptoms) (54\%) [13]. Furthermore, for HS, Facebook Groups can be surveyed for effective pain control therapies [14] or diets [15] posted by patients. Nevertheless, the psychosocial benefit of being able to connect with others who are undergoing a similar journey can be therapeutic for many patients, more so than the medical advice shared among its group members.

As mentioned previously, Facebook has become a source of disease-related information for users experiencing skin conditions. However, as all users have the freedom to create and propagate posts of their choosing, this content can easily be misleading or false. This could lead to unfortunate and potentially harmful consequences for users sharing treatment ideas that are not supported by scientific evidence or peer-reviewed approvals [16]. Possibly adding to this issue is Facebook's heavy utilization of the user-directed advertisements. For example, 56-95\% of recent Facebook posts from top hair loss pages advertised hair loss products and hair restoration clinics [17]. Of this content, only 3-13\% contained health information supported by a medical or science professional or cited scientific publications. Fundraising for private alopecia organizations also seems to be a focus of several Facebook Groups [17]. Fortunately, to combat the spread of misinformation, Facebook has begun implementing third-party fact-checking software and networks of trained fact-checkers [18]. While efforts have been focused on information about elections and COVID-19, factchecking processes hold promise for verifying the accuracy of dermatologic information spread to Facebook users.

\section{Twitter and Dermatology}

Founded in 2006, Twitter has become a popular social media platform for students, residents, and physicians to communicate and contribute to discussions about a variety of dermatologic topics. There are currently 199 million daily active users on Twitter [19]. Twitter allows users to create and share their own text, photo, and video posts in brief short-format "tweets" limited to 280 characters [20]. They can also "favorite" posts made by other users and reply to posts as well. Users can "retweet" to re-share content that was posted by another user. Twitter encourages users to "mention" other Twitter accounts when creating posts, in order to notify another person about their tweet and prompt them to respond. The "hashtag" function permits users to highlight posts for others who are interested in the same topic and allow content categorized by hashtag terms to be easily searchable and shareable. Users can also "follow" accounts to view their content on their personal feed [21].

Dermatology and dermatologists on Twitter have been popularized in several ways. Users have leveraged Twitter to promote dermatology clinics and healthcare practices, share research and personal experiences, and engage with professional and patient-centered organizations. The JAAD, JAMA Dermatology, and other dermatology journals have active Twitter accounts to announce publications of newly conducted research [22]. Furthermore, Twitter discussions cover a wide variety of dermatology topics, including users' sun-related and personal skin cancer experiences, with the goal of placing a strong emphasis on health promotion [23]. Real-time trends in health issues and public health have been identified by monitoring the content of tweets [24]. Professional dermatology organizations, such as the dermRounds Dermatology Network (@ dermatology, with 18,600 followers as of July 2021), manage active, highly followed Twitter accounts. Through frequent posts, interested users can connect with each other and debate answers to dermatology clinical photo quizzes. Thriving online journal clubs including hundreds of physicians around the world have been established on Twitter, to provide real-time monthly meetups for networking and exchanging content and ideas [25, 26]. Large patient-oriented non-profit organizations such as the National Psoriasis Foundation are also significant bases of knowledge and support for patients with chronic dermatologic conditions [27]. Lastly, advertisements on social media sites such as Twitter have shown to be very influential in increasing patient volume compared to other forms of advertising, such as television or print [2].

There is clearly a great benefit in Twitter's ability to quickly and easily share information, build connections with others, and foster professional relationships. However, flaws also exist with the usage of this and other social media platforms. Spread of misinformation, especially about topics such as inaccurate or ineffective treatments for dermatologic conditions, can easily be done on Twitter due to the lack of monitoring and no accuracy verification of users' tweets. A recent study of how acne is discussed on Twitter highlighted extreme discrepancies and misperceptions relative to AAD 
recommendations. Incorrect information and unconventional home remedies were common in Twitter content [28]. While a user's authentic identity can be proven through Twitter's verification process, this is not required to establish an account and anyone (for example) can claim to be a boardcertified dermatologist without providing credentials. The spread of misleading information can have the unfortunate consequence of leading to ill-fated, if not harmful, results for patients who rely on Twitter for medical advice rather than seeking professional care. Fortunately, the increasing presence of board-certified dermatologists on Twitter could promote the distribution of accurate information to users.

\section{Instagram and Dermatology}

Initially launched in 2010, the utilization of Instagram has steadily increased since its introduction to the world of social media. Although less popular than Facebook, Instagram now has over 1 billion active monthly users and 500 million daily users worldwide [29, 30]. Approximately $40 \%$ of the US population reports using Instagram, an increase from around $30 \%$ in 2017 [31]. As a free social media platform, Instagram content is shared as either a photo, video, Story (visible to followers of the user's account for $24 \mathrm{~h}$ ), or Reel (short video clips with creative audio and special effects up to $30 \mathrm{~s}$ long). Instagram users can interact with other users through direct messaging, commenting on posts, or liking posts [32]. While users are limited to a 2200 character count when posting on Instagram, the length of a post is much more extensive than that allowed by Twitter, which is only 280 characters [33]. Although not unique to Instagram, short-phrase or compound-word "hashtags" are often added to be used as subjects for the post, automatically providing a searchable link to related posts with the same "hashtag" [34]. This feature can be helpful for users when connecting with one another through similar posts and topics.

Given its popularity and wide reach, Instagram has been used as a means for acquiring and disseminating healthrelated information, as well as a tool for physicians to connect with patients and other physicians or colleagues. Hospitals and healthcare workers are increasingly engaging with social media, and this eruption in physician engagement has undoubtedly created unprecedented opportunities for physicians to network with one another. Given the visual nature of Instagram, it is unsurprising that dermatology has a substantial presence on the platform; topics range from educational to promotional while using hashtags to connect ideas. The most popular dermatology hashtags in one recent study included \#acne, \#alopecia, and \#eczema [35]. Perhaps due to Instagram's effectiveness at rapidly disseminating information to a large network of users, studies have found that dermatology patients and trainees are increasingly turning to Instagram as an educational platform [36]. For example, there has been a substantial increase in the presence of US dermatology residency programs on Instagram, from only 9 programs in 2019 having active Instagram accounts [9] to 74 programs in early 2021 [10, 37]. Of the followers for these accounts, the majority is the general population (66\%) followed by medical students $(10 \%)$, attendings (10\%), dermatology programs (8.5\%), and residents/fellows (5.8\%) [10].

While more physicians are using social media to educate and engage in patient outreach, the platform has been prone to a flood of misinformation presented by non-healthcare providers, likely related to the prevalence of commercial content from businesses and vendors on Instagram. A significant number of Instagram posts are promotional or advertising [38]. Before-and-after photos and advertisements for cosmetic procedures are often interspersed with informational material for patients and academic announcements [35]. Furthermore, a study of dermatology-related hashtags revealed that only $5 \%$ of 146 unique influencers identified were dermatologists, and a similar analysis of top Instagram dermatology posts indicated that only $35 \%$ of unique posters were healthcare workers [39]. Corroborating this, a study of skin of color-related hashtags on Instagram concluded that non-dermatologists were responsible for the majority of posts, with dermatologists generating only $12 \%$ of top content [40]. Most non-dermatologist influencers (and also some dermatologists) promoted products and services on Instagram, which may create conflicts of interest [41]. A lack of educational content backed by board-certified dermatologists may be detrimental, as inaccurate sources may contribute to the spread of misinformation.

\section{TikTok and Dermatology}

TikTok is a video-sharing social media app created in 2016 [42], and one of the fastest-growing with over 1 billion monthly active users and 2 billion downloads since its launch worldwide [43]. Short videos between 5 and $60 \mathrm{~s}$ long are uploaded by users with captions and hashtags to reach audiences interested in similar topics. Videos can be "duetted" by other users, a function that allows videos to be played alongside (adjacent to) a new video being posted. Similarly, users can "reply" to videos and link the prior video in their caption. Videos can also garner "hearts" and comments from other users and can be shared with others if the feature is allowed by the content creator. User interactions with videos contribute to a personalized "For You" page, where TikTok displays similar videos tailored to the user's interests.

Dermatology topics are a growing presence within TikTok, totaling 4.5 billion views for the hashtag \#acne and 633.8 million for \#acnetreatment. Other popular TikTok 
hashtags include \#eczema, \#psoriasis, and \#hidradenitissuppurativa [42]. Content creators have been identified as patients, board-certified dermatologists, and estheticians, discussing everything from medically accurate educational videos to natural remedies attempted at home [44]. Several board-certified dermatologists now use TikTok in an effort to spread medically accurate information and dispel misinformation being shared by other users [42]. Another form of TikTok content includes "challenges," which are viral video trends that encourage others to create similar content. This has proven to be a source of potentially unsafe behavior, with examples ranging from the "\#tanningbedchallenge," in which users post videos from within tanning beds, to the "\#sunburnchallenge," which assembles a collection of drastically sunburned participants of the challenge [45].

As with other forms of social media, the accuracy and reliability of content shared in TikTok videos are clearly questionable. Influencers are able to spread misinformation to a large population of users that may believe in the accuracy of the content displayed due to the creator's increased following, which can have unfortunate consequences. However, possible avenues such as the creation of verified TikTok accounts by official entities such as the AAD could help dispel misinformation, similar to the efforts from an increasing number of dermatologists on TikTok [43]. TikTok's unique "duet" feature could be particularly advantageous relative to other platforms, since it allows dermatologists a real-time opportunity to correct inaccurate content before further spread $[38,46]$.

\section{YouTube and Dermatology}

YouTube is a platform for video-sharing allowing users to upload videos and live streams, vote and comment on content, and subscribe to their favorite content creators via channels. As of mid-2021, YouTube features over 2 billion users - almost one-third of the total internet - watching over 1 billion hours of video daily, spanning 100 countries and 80 languages [47]. Increasingly, YouTube is a popular resource for patients and the general public seeking information about their health and medical advice. It is estimated that $80 \%$ of Internet users search for health information online, and YouTube ranks as the second-most accessed website worldwide. In particular, patients with chronic illness depend on internet-based health resources such as YouTube [48].

In dermatology, YouTube has been an instrumental tool for the evaluation of emerging trends and content. To better understand what patients are seeing on YouTube and potentially improve health outcomes, recent studies have found that almost half of dermatology YouTube videos were uploaded by or featured by a healthcare professional (many were board-certified dermatologists) and that educational videos were the most prevalent. Content type has shifted towards more educational and personal videos, with decreases in advertising and promotional content [49].

However, the level of accuracy and misinformation in dermatology-related YouTube videos varies greatly. For example, one study analyzing the quality of YouTube videos about topical treatments for psoriasis found that natural therapies were heavily favored, instead of treatments recommended by the AAD [50]. Most videos were financially biased, and the vast majority did not feature a healthcare provider. Furthermore, patient testimonials triggered the most views and user interaction, whereas videos featuring healthcare providers had the lowest user engagement metrics. Another study demonstrated that almost two-thirds of the top 100 most-viewed psoriasis YouTube videos depicted misleading or even dangerous content. Poor-quality videos received the highest proportions of positive ratings, while the opposite was observed for low-quality videos [48]. Similarly, YouTube videos contained substantial false or misleading information on tanning beds $(40 \%$ of videos analyzed) and sunscreen (20\%), and only about $60 \%$ of videos mentioned the negative effects of ultraviolet light exposure [51]. Dermatologists and professional dermatological organizations should be encouraged to monitor and generate increased volumes of evidence-based YouTube content, in order to improve patient-directed education and outreach through the platform.

\section{Snapchat and Dermatology}

Snapchat is another popular social media application, created in 2011. This application allows short "Snaps" (less than 10-s duration clips) of pictures and videos to be shared between its users. Instant messaging, video chat, trophies, and user-created avatars are appealing features of Snapchat, as well as the ephemeral impulsive nature of Snaps where transmitted content is designed to disappear from the platform once viewed or opened by the intended audience. There are estimated to be 280 million users on Snapchat, with over 5 billion Snaps created daily [52]. What sets this application apart from traditional video and photo sharing are the unique visual modification options known as "filters." To dermatologists, the most impactful and concerning features may be the effects from Snapchat beautification filters, which heightens or exaggerate the users' appearance. These filters have even been a driving force for some of its users to pursue cosmetic modifications. This phenomenon known as "Snapchat dysmorphia" has led to patient encounters with plastic surgeons, and likely dermatologists, singularly focused on cosmesis to resemble these filtered images [53]. Understanding the basis of these requests may help guide clinicians during these encounters, as these may be 
particularly challenging or unrealistic expectations to meet. Additionally, Snapchat may be impacting its users through its advertisements, which have been incorporated into filters and "Snapchat Stories." Stories allow users to flip through magazine-like collections of popular news items and celebrities. Some fields in medicine have used this application to their advantage for self-promotion, communication, and education with their audience; however, dermatology has yet to create a notable presence on Snapchat [3]. Conversely, plastic surgeon influencers have been highly publicized and impactful. Celebrity plastic surgeons Dr. Michael Salzhauer (Snapchat username @ therealdrmiami) and Dr. Matthew Schulman (@nycplasticsurg) regularly receive over 1 million views per Snap, while Dr. Sandra Lee (@DrPimplePopper), a well-known dermatologist social media influencer and television personality, recently lacked an estimation of Snapchat views per day (usually an indicator of low view count). Additionally, dermatological journals, organizations, or patient support groups currently do not have Snapchat accounts. This may signify that there is further potential for dermatology's expansion on this platform [54]. Due to Snapchat's 24-hr post expiration and time limits for viewing meaningful and lasting content, Snapchat could be less favorable in comparison with other social media platforms for education and advocacy.

\section{Other Social Media Applications and Dermatology}

The dynamic ever-evolving landscape of social media applications entails that new and old platforms can wax and wane in popularity and purpose. Reddit is one of the fastest growing online communities in 2020, with 430 million total users with 52 million daily active users (up 44\% from 2019) and 303 million posts (up 52\% from 2019) [55]. Sub-communities of Reddit, or "subreddits," are usergenerated forums for the posting of ideas, questions for the community, and links to news articles, images, or videos for commentary. Users can therefore focus on information targeted to their interests and up-vote or down-vote posts and comments to increase the visibility of the content. In 2020 , the subreddit " $r$ /coronavirus" became the largest online community dedicated to COVID-19 (and by activity the \#3 top-growing online community in 2020 overall). Reddit moderated the discussion and ensured propagation of only accurate fact-checked information on the subreddit [56]. Reddit's fact-checking may be beneficial for dermatology in the future, as several highly active dermatologyrelated subreddits exist. Since the sharing of cosmetic advice, identification of skin conditions, and medication recommendations are common on these subreddits, misinformation can be propagated as users are freely allowed to post responses. Dermatologists should recognize Reddit trends and perhaps self-identify on Reddit forums to highlight inaccuracies early [57].

Messaging applications such as WhatsApp, Facebook Messenger, WeChat, and Line also have myriad applications in healthcare. Chat-based hotlines can connect users with healthcare professionals in real time and could have potential in crisis settings where other communications may be limited in a rapidly changing situation [58]. Since photos and video can be transmitted through instant messaging, dermatology teleconsultations through messaging applications have been piloted and are well-received by patients [59]. However, a lack of messaging app security may introduce privacy risks. Dermatologists may receive messages seeking advice about skin lesions from friends, family, or others without a prior patient-physician relationship. While some apps support end-to-end encryption (e.g., WhatsApp), others may have a variable password and access controls, inability to audit information, or track actions through the app. Especially given the current shift to telehealth, Health Insurance Portability and Accountability Act (HIPAA) compliance should be considered before implementing apps for healthcare delivery [3].

\section{Conclusion}

Almost half the world's population uses social media, and this number will only continue to grow [2]. Our overview of apps establishes that healthcare- and dermatology-related social media usage is extremely popular, creating opportunities for research, outreach, and even tracking trends in public health. Dermatologists must be aware of the interplay between social media and healthcare, and should be compelled to develop a social media presence - not only for networking and personal benefits, but also to combat inaccurate information and disseminate sound evidence-based medical knowledge. While each social media platform can operate independently, maintaining a presence on more than one of the discussed apps may be beneficial to reach a wider audience. For instance, dermatology TikTok videos can be cross-posted to Instagram Reels to potentially boost engagement with the content presented [38]. However, privacy concerns and potential biases also exist, with social media users generally being younger and female with access to the Internet and mobile devices [60]. Despite this, the utility of social media apps in promoting dermatology, providing health education, and fostering professional networking is highly influential and should be explored. 


\section{Compliance with Ethical Standards}

Human and Animal Rights and Informed Consent This article does not contain any studies with human or animal subjects performed by any of the authors.

Conflict of Interest There are no conflicts of interest to declare from any of the authors.

\section{References}

1. Limaye RJ, Sauer M, Ali J, Bernstein J, Wahl B, Barnhill A, et al. Building trust while influencing online COVID-19 content in the social media world. Lancet Digit Health. 2020;2(6):e277-8. https://doi.org/10.1016/s2589-7500(20)30084-4.

2. DeBord LC, Patel V, Braun TL, Dao H Jr. Social media in dermatology: clinical relevance, academic value, and trends across platforms. J Dermatolog Treat. 2019;30(5):511-8. https://doi.org/ $10.1080 / 09546634.2018 .1530444$. This article was a thorough and comphrensive overview of social media use in dermatology through the mutliple different platforms. it contributed much of the baseline data regarding number of users per social media site. Additionally, it examines social media use in education and outreach in the community as well as trends seen across each site and its content creators.

3. Laughter M, Zangara T, Maymone M, Rundle C, Dunnick C, Hugh J, et al. Social media use in dermatology. Dermatol Sin. 2020;38(1):28-34. https://doi.org/10.4103/ds.ds_43_19.

4. Sierro TJ, Young PM, Kassabian SK, Wu KK, Armstrong AW. Dermatologists in social media: a study on top influencers, posts, and user engagement. J Am Acad Dermatol. 2020;83(5):1452-5. https://doi.org/10.1016/j.jaad.2020.03.001.

5. Damanpour S, Nazarian R, Deutsch A, Hosgood HD, Kim J, McLellan BN. Social media activity is associated with higher physician ratings by patients. J Am Acad Dermatol. 2021;84(5):14558. https://doi.org/10.1016/j.jaad.2020.06.1015.

6. Facebook Reports First Quarter 2021 Results. https://investor.fb. com/investor-news/press-release-details/2021/Facebook-ReportsFirst-Quarter-2021-Results/default.aspx. Accessed 15 July 2021.

7. Kim W, Vender R. Use of Facebook as a tool for knowledge dissemination in dermatology. J Cutan Med Surg. 2014;18(5):341-4. https://doi.org/10.2310/7750.2014.14022.

8. Facebook: American Academy of Dermatology. https://www. facebook.com/AADskin/. Accessed 15 July 2021.

9. St Claire KM, Rietcheck HR, Patel RR, Dellavalle RP. An assessment of social media usage by dermatology residency programs. Dermatol Online J. 2019;25(1).

10. Yong-hun Kim, Ali NS, Vidal NY. Social media use in residency recruitment during the COVID-19 pandemic. Dermatology Online Journal. 2021;27(6):1-3.

11. Guckian J, Jobling K, Oliphant T, Weatherhead S, Blasdale K. 'I saw it on Facebook!' Assessing the influence of social media on patient presentation to a melanoma screening clinic. Clin Exp Dermatol. 2020;45(3):295-301. https://doi.org/10.1111/ced. 14100.

12. Petukhova TA, Wilson BN, Gadjiko M, Lee EH, Wang J, Rossi $\mathrm{AM}$, et al. Utilization of Facebook for support and education by patients with skin cancer. Dermatol Online J. 2020;26(3).

13. Golbari NM, Porter ML, Kimball AB. Online communications among hidradenitis suppurativa patients reflect community needs. J Am Acad Dermatol. 2019;80(6):1760-2. https://doi.org/10. 1016/j.jaad.2018.08.031.
14. Fernandez JM, Thompson AM, Borgstrom M, Orenstein LAV, Hsiao JL, Shi VY. Pain management modalities for hidradenitis suppurativa: a patient survey. J Dermatolog Treat. 2020:14. https://doi.org/10.1080/09546634.2020.1822501.

15. Fernandez JM, Marr KD, Hendricks AJ, Price KN, Ludwig CM, Maarouf M, et al. Alleviating and exacerbating foods in hidradenitis suppurativa. Dermatol Ther. 2020;33(6):e14246. https://doi. org/10.1111/dth.14246.

16. Schuster B, Ziehfreund S, Biedermann T, Zink A. Psoriasis 2.0: Facebook as a source of disease-related information for patients with psoriasis. J Dtsch Dermatol Ges. 2020;18(6):571-81. https:// doi.org/10.1111/ddg.14070.

17. Gupta AK, Ivanova IA. Hair loss treatment information on Facebook: content analysis and comparison with other online sources. J Cosmet Dermatol. 2021;20(2):577-83. https://doi.org/10.1111/ jocd.13830. This article highlighted the type of content found in dermatology Facebook Goups, specifically in hair loss and alopecia groups. It showed that most of the value and use of these groups was centred around peer support, raising awareness, sharing experiences, but lacked hair loss treatment information. It also pointed out how patient would prefer to read educaitonal material written by a physican rather than a layperson online opening more future opportunties for dermatologists to interact with patient populations via Facebook.

18. How Facebook's third-party fact-checking program works. https:// www.facebook.com/journalismproject/programs/third-party-factchecking/how-it-works. Accessed 16 July 2021.

19. Twitter Q1 2021 Letter to Shareholders. https://s22.q4cdn.com/ 826641620/files/doc_financials/2021/q1/Q1'21-Shareholder-Letter.pdf. Accessed 15 July 2021.

20. Rosen A, Ihara I: Giving you more characters to express yourself. https://blog.twitter.com/official/en_us/topics/product/2017/Giving-you-more-characters-to-express-yourself.html. Accessed 15 July 2021.

21. Twitter Help Center Glossary. https://help.twitter.com/en/resources/ glossary. Accessed 15 July 2021

22. Patel RR, Hill MK, Smith MK, Seeker P, Dellavalle RP. An updated assessment of social media usage by dermatology journals and organizations. Dermatol Online J. 2018;24(2).

23. Vasconcelos Silva C, Jayasinghe D, Janda M. What can Twitter tell us about skin cancer Communication and prevention on social media? Dermatology. 2020;236(2):81-9. https://doi.org/10.1159/ 000506458.

24. Sarker A, Magge A, Sharma A. Dermatologic concerns communicated through Twitter. Int J Dermatol. 2017;56(8):e162-3. https:// doi.org/10.1111/ijd.13506.

25. Gottesman SP, Klein WM, Hosler GA, Veprauskas KR, Rush PS, Gardner JM. \#dermpathJC: the first online dermatopathology Twitter journal club. J Cutan Pathol. 2018;45(5):370-3. https:// doi.org/10.1111/cup.13110.

26. Daneshjou R, Adamson AS. Twitter journal clubs: medical education in the era of social media. JAMA Dermatol. 2020;156(7):729-30. https://doi.org/10.1001/jamadermatol.2020. 0315.

27. Amir M, Sampson BP, Endly D, Tamai JM, Henley J, Brewer AC, et al. Social networking sites: emerging and essential tools for communication in dermatology. JAMA Dermatol. 2014;150(1):56-60. https://doi.org/10.1001/jamadermatol.2013.6340.

28. Shive M, Bhatt M, Cantino A, Kvedar J, Jethwani K. Perspectives on acne: what Twitter can teach health care providers. JAMA Dermatol. 2013;149(5):621-2. https://doi.org/10.1001/jamadermatol. 2013.248. This article contributed to the quality and accuracy of dermatologic content produced via "tweets"on Twitter. It use word frequency comparisons to $\mathrm{AAD}$ and Twitter to find many discrepancies in medical advice or content produced, especially in regards to acne counseling. 
29. Instagram: about us. https://about.instagram.com/about-us. Accessed 15 July 2021.

30. Instagram Statistics, User Counts and Facts (2021). https:// expandedramblings.com/index.php/important-instagram-stats/. Accessed 15 July 2021.

31. Pew Research Center: Growing share of Americans say they use YouTube; Facebook remains one of the most widely used online platforms among U.S. adults. https://www.pewresearch. org/internet/2021/04/07/social-media-use-in-2021/pi_2021-0407_social-media_0-01/. Accessed 15 July 2021.

32. Instagram: Features - our features. https://about.instagram.com/ features. Accessed 15 July 2021.

33. Jackson D: Know your limit: the ideal length of every social media post. https://sproutsocial.com/insights/social-media-charactercounter/. Accessed 15 July 2021.

34. Karimkhani C, Connett J, Boyers L, Quest T, Dellavalle RP. Dermatology on Instagram. Dermatol Online J. 2014;20(7).

35. Braunberger T, Mounessa J, Rudningen K, Dunnick CA, Dellavalle RP. Global skin diseases on Instagram hashtags. Dermatol Online J. 2017;23(5).

36. Chen JY, Gardner JM, Chen SC, McMichael JR. Instagram for dermatology education. J Am Acad Dermatol. 2020;83(4):11756. https://doi.org/10.1016/j.jaad.2020.02.001.

37. Schwartzman G, Qureshi A, Friedman AJ. Utilization of Instagram by dermatology residency programs in the era of COVID19. J Am Acad Dermatol. 2021;85(1):204-6. https://doi.org/10. 1016/j.jaad.2021.03.078.

38. Szeto MD, Presley CL, Pulsipher KJ, Harp T, Rundle CW, Sivesind TE, et al. Dermatologist influencers on social media: Instagram Reels and TikTok interactive short videos. J Am Acad Dermatol. https://doi.org/10.1016/j.jaad.2021.04.052.

39. Park JH, Christman MP, Linos E, Rieder EA. Dermatology on Instagram: an analysis of hashtags. J Drugs Dermatol. 2018;17(4):482-4.

40. Wells TM, Rundle CW, Szeto MD, Presley C, Dellavalle RP. An analysis of skin of color dermatology related content on Instagram. J Drugs Dermatol. 2020;19(7):746-54. https://doi.org/10. 36849/jdd.2020.5142.

41. Ranpariya V, Chu B, Fathy R, Lipoff JB. Dermatology without dermatologists? Analyzing Instagram influencers with dermatologyrelated hashtags. J Am Acad Dermatol. 2020;83(6):1840-2. https:// doi.org/10.1016/j.jaad.2020.05.039. Important resource that illuminates the authors and credentials of many influencial Instagram accounts that are creating dermatology-related content. It was this article that showed that only $38 \%$ of these posts and accounts were from healthcare professionals and but less $(\sim 4 \%)$ of accounts were run by board-certified dermatologists.

42. Roche L, Nic Dhonncha E, Murphy M. TikTok ${ }^{\mathrm{TM}}$ and dermatology: promises and pearls. Clin Exp Dermatol. 2021;46(4):7379. https://doi.org/10.1111/ced.14529.

43. Zheng DX, Mulligan KM, Scott JF. TikTok and dermatology: an opportunity for public health engagement. J Am Acad Dermatol. 2021;85(1):e25-6. https://doi.org/10.1016/j.jaad.2021.02.050.

44. Villa-Ruiz C, Kassamali B, Mazori DR, Min M, Cobos G, LaChance A. Overview of TikTok's most viewed dermatologic content and assessment of its reliability. J Am Acad Dermatol. 2021;85(1):273-4. https://doi.org/10.1016/j.jaad.2020.12.028.

45. De La Garza H, Maymone MBC, Vashi NA. Impact of social media on skin cancer prevention. Int J Environ Res Public Health. 2021;18(9). https://doi.org/10.3390/ijerph18095002.
46. Presley CL, Pulsipher KJ, Rietcheck HR, Szeto MD, Laughter MR, Dellavalle RP. Reply to "Dermatologists in social media: a study on top influencers, posts, and user engagement": dermatologist influencers on TikTok. J Am Acad Dermatol. 2021. https:// doi.org/10.1016/j.jaad.2021.01.090.

47. YouTube for Press. https://www.youtube.com/intl/en-GB/about/ press/. Accessed 16 July 2021.

48. Mueller SM, Jungo P, Cajacob L, Schwegler S, Itin P, Brandt $\mathrm{O}$. The absence of evidence is evidence of non-sense: crosssectional study on the quality of psoriasis-related videos on YouTube and their reception by health seekers. J Med Internet Res. 2019;21(1):e11935. https://doi.org/10.2196/11935.

49. St Claire KM, Rietcheck HR, Patel RR, Dunnick C, Dellavalle RP. Dermatology on YouTube - an update and analysis of new trends. Dermatol Online J. 2018;24(12).

50. Pithadia DJ, Reynolds KA, Lee EB, Wu JJ. A cross-sectional study of YouTube videos as a source of patient information about topical psoriasis therapies. J Dermatolog Treat. 2020;31(4):366-9. https://doi.org/10.1080/09546634.2019.1597247.

51. Ruppert L, Køster B, Siegert AM, Cop C, Boyers L, Karimkhani $\mathrm{C}$, et al. YouTube as a source of health information: analysis of sun protection and skin cancer prevention related issues. Dermatol Online J. 2017;23(1).

52. Sultan M, Brown EM, Thomas RH. Clinicians embracing social media: potential and pitfalls. Epilepsy Behav. 2021;115: 106462. https://doi.org/10.1016/j.yebeh.2019.106462.

53. Ramphul K, Mejias SG. Is "Snapchat dysmorphia" a real issue? Cureus. 2018;10(3): e2263. https://doi.org/10.7759/cureus.2263.

54. Patel RR, Yazd NKK, Dellavalle RP. Dermatology on Snapchat. Dermatol Online J. 2017;23(7).

55. Reddit's 2020 Year in Review. https://redditblog.com/2020/12/ 08/reddits-2020-year-in-review/. Accessed 16 July 2021.

56. Reddit's 2020 Year in Review - Community Reflections. https://redditblog.com/2020/12/08/reddits-2020-year-in-reviewcommunity-reflections/. Accessed 16 July 2021.

57. Buntinx-Krieg T, Caravaglio J, Domozych R, Dellavalle RP. Dermatology on Reddit: elucidating trends in dermatologic communications on the world wide web. Dermatol Online J. 2017;23(7). Crucial resouce highlighting the different Reddit pages affiliated with dermatologic diseases and number of associated members. Additionally, this was an important source due to the lack of overall articles focusing on Reddit prior to this. This paper contributes data revealing the use and content of dermatologic diseases pages via Reddit.

58. Brody C, Star A, Tran J. Chat-based hotlines for health promotion: a systematic review. Mhealth. 2020;6:36. https://doi.org/10.21037/ mhealth-2019-di-13.

59. Wang YC, Ganzorig B, Wu CC, Iqbal U, Khan HA, Hsieh WS, et al. Patient satisfaction with dermatology teleconsultation by using MedX. Comput Methods Programs Biomed. 2018;167:3742. https://doi.org/10.1016/j.cmpb.2018.10.015.

60. Sadah SA, Shahbazi M, Wiley MT, Hristidis V. A study of the demographics of web-based health-related social media users. J Med Internet Res. 2015;17(8):e194. https://doi.org/10.2196/jmir. 4308.

Publisher's Note Springer Nature remains neutral with regard to jurisdictional claims in published maps and institutional affiliations. 